\title{
Endoscopic Appearance of a Duodenal Infection by Mycobacterium avium-intracellulare in AIDS
}

We report here the case of a 37-year-old woman, previously a parenteral drug abuser, with HIV infection diagnosed four years before, who presented with fever, weight loss, severe dysphagia and abdominal pain. Endoscopy showed thickening of the mucosal folds and multiple yellow-whitish nodules in the duodenum, also involving the bulb (Figure 1). A diagnosis of infection by $\mathrm{Myco}$ bacterium avium-intracellulare (MAI) was established (positive Haemoccults and cultures of samples obtained from the antral and duodenal mucosa). The pathological study of the biopsy samples obtained from the duodenum led to the diagnosis of infection by MAI, with a light-microscopic appearance similar to that in Whipple's disease. A scanning electron-microscopic study was performed, and the most significant finding was visualization of the electrolucent capsule of the rods (Figure 2).

The endoscopic lesions caused by MAI infections have been described as "minute superficial ulcerations throughout the duodenum" (2) or "multiple, somewhat raised, yellow lesions affecting the duodenum" (3). Our patient showed lesions (several yellow-white nodules) that were similar to those described in Whipple's disease (4) and to those described by Vázquez-Iglesias et al. (3) in MAI infection, except that the lesions were already evident from the beginning of the duodenal bulb, and there were no confluent lesions in the second portion of the duodenum. In addition, in our case, a few antral erosions were seen, which could be similar to the ones described in Whipple's disease (4); although MAI was detected in the culture, the presence of histological lesions caused by MAI could not be confirmed in the pathological study of the samples.

Various reports have concluded that AIDS enteropathy may mimic Whipple's disease (4). Our patient also presented with an MAI infection that had the optical appearance of Whipple's disease. Both conditions produce erythematous macular lesions in the small intestine, with villous widening and foamy macrophages which, using the periodic acid - Schiff reaction method, show the presence of bacillary structures called Sieracky's structures in Whipple's disease (2). The differential diagnosis is made using Ziehl's technique, which is negative in Whipple's disease and positive in MAI infection. In the latter case, the rods are generally intact and have an electrolucent capsule, while in Whipple's disease they appear at different stages of intracellular digestion, have a dense capsule, are Gram-positive, suffer binary fission, and disappear under antibiotic treatment (5).

O. Bosch ', J. C. Porres ', G. Martinez Quesada ${ }^{2}$, H. Oliva $^{3}$, C. González Campos ${ }^{1}$

${ }^{1}$ Department of Gastroenterology, ${ }^{2}$ Department of Internal Medicine, and ${ }^{3}$ Department of Pathology, Fundación Jiménez Díaz, Universidad Autónoma de Madrid, Madrid, Spain

\section{References}

1 Gillian JS, Urmacher $C$, West $R$, et al.: Disseminated mycobacterium avium-intracellulare infections in acquired immunodeficiency syndrome mimicking Whipple's disease. Gastroenterology 1983; 85: 1187.

2 Roth RI, Owen RL, Keren DF, et al.: Intestinal infection with Mycobacterium avium in acquired immune deficiency syndrome (AIDS):

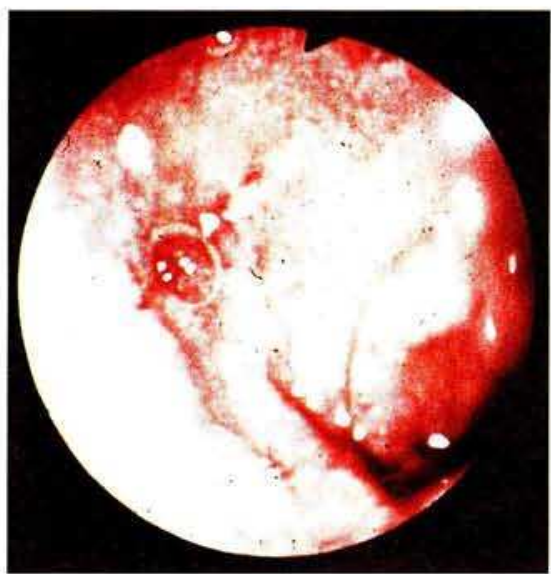

Figure 1:

Duodenal bulb with thickened mucosal folds and several elevated yellow-white nodules, $1-4 \mathrm{~mm}$ in diameter. The second portion of the duodenum was not involved by confluent lesions.

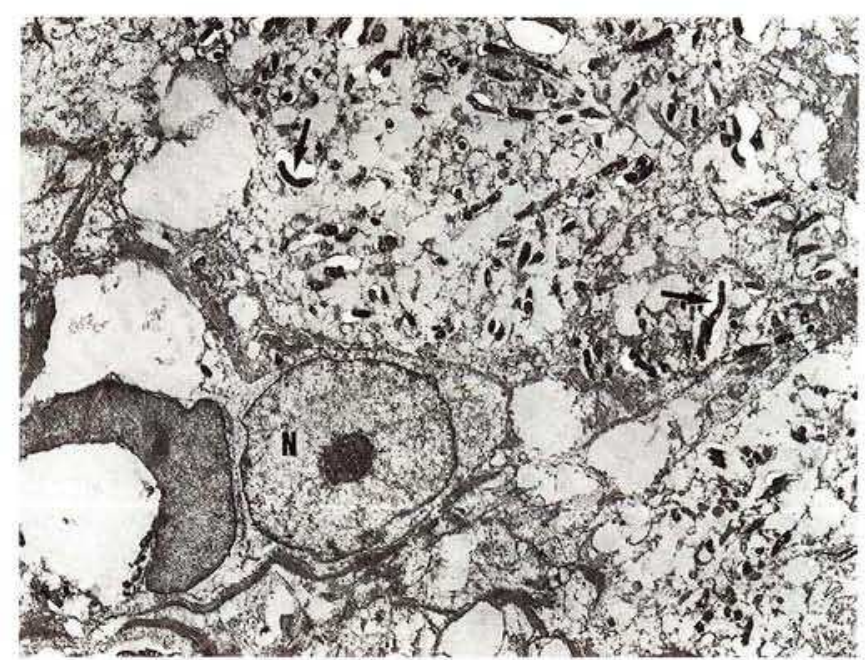

Figure 2: Ultrastructure of a histiocyte, with numerous intracytoplasmic bacilli, showing the electrolucent capsule of the rods $(\mathrm{EM} \times 5000)$. $\mathrm{N}$ : nucleus of the histiocyte. Arrow: intracytoplasmic bacili.

histological and clinical comparison with Whipple's disease. Dig. Dis. Sci. 1985; 30: 497-504.

3 Vazquez-Iglesias JL, Yañez, Durana J, et al.: Infection by Mycobacterium avium-intracellulare in AIDS: endoscopic duodenal appearance mimicking Whipple's disease. Endoscopy 1988; 20: 279-280.

4 Geboes K, Ectors N, Heidbuchel H, et al.: Whipple's disease: endoscopic aspects before and after therapy. Gastrointest. Endosc. 1990; 36: $247-252$.

5 Dobbins WO, Kawanishi H: Bacillary characteristics in Whipple's disease: an electron-microscopic study. Gastroenterology 1981; 80: $1468-1475$.

Corresponding Author

C. González Campos, M.D.

Department of Gastroenterology

Fundación Jiménez Diaz

Avenida Reyes Católicos 2

28040 Madrid

Spain 